\title{
Review
}

\section{Puerarin-an isoflavone with beneficial effects on bone health}

\author{
Bartosz Kulczyński ${ }^{1}$, Anna Gramza-Michałowska ${ }^{1, *}$, Joanna Suliburska ${ }^{2}$, Andrzej Sidor ${ }^{1}$ \\ ${ }^{1}$ Department of Gastronomy Sciences and Functional Foods, Faculty of Food Science and Nutrition, Poznań University \\ of Life Sciences, 60-624 Poznań, Poland, ${ }^{2}$ Department of Human Nutrition and Dietetics, Faculty of Food Science and \\ Nutrition, Poznań University of Life Sciences, 60-624 Poznań, Poland
}

\section{TABLE OF CONTENTS}

\author{
1. Abstract \\ 2. Introduction \\ 3. Effect of puerarin on bone health \\ 3.1 In vitro studies \\ 3.2 Animal model studies \\ 4. Summary and perspective \\ 5. Author contributions \\ 6. Ethics approval and consent to participate \\ 7. Acknowledgment \\ 8. Funding \\ 9. Conflict of interest \\ 10. References
}

\section{Abstract}

Puerarin is a compound from the group of isoflavones, naturally occurring in plants of the genus Pueraria, whose representatives include, among others, Pueraria lobata and Pueraria mirifica. Relatively many scientific studies on the biological activity of puerarin have been conducted so far. It seems that most attention was paid to the effect of puerarin on bone health. However, until now, no published studies have been collected and discussed in that regard. Based on the available data obtained from in vitro studies and on the animal model, it can be clearly shown that puerarin is an effective compound in inhibiting bone resorption and improving bone structure. Consumption of puerarin may be associated with the prevention of bone mass loss and thus can reduce the risk of developing osteoporosis. However, it is necessary to conduct human intervention studies to confirm the effectiveness of such action.

\section{Introduction}

Osteoporosis is a metabolic bone disease associated with reduced bone mass as well as increased degradation of bone microarchitecture. As a result, it is characterized by reduced bone strength and increased risk of frac- tures [1]. Osteoporosis is a major public health challenge worldwide [2]. It is estimated that in 2010 osteoporotic fractures caused 43,000 deaths in the European Union alone [3]. As for the USA, nearly 54 million Americans have too low bone mass and show an increased risk of fractures [4]. In addition, more than 8.9 million fractures per year are reported worldwide. The majority of these fractures concern the European population (34.8\%) [1]. Therefore, it seems necessary to take all measures to prevent this disease. The most important, modifiable risk factors for osteoporosis include lack of physical activity, smoking and improper nutrition, mainly related to the deficiency of nutrients essential for bone structure and homeostasis, including vitamin D and calcium [5]. However, a number of other compounds present in the diet are also significant for bone health. These include vitamin C [6], potassium, magnesium, omega-3 fatty acids [7], carotenoids (e.g., lycopene, beta-carotene), polyphenols [8]. A group of phytochemicals particularly well-studied in terms of their effect on bone health includes soy isoflavones, including, above all, genistein and daidzein [9]. However, there are many other compounds among isoflavones which are derived not only from soya. One of isoflavones, puerarin (daidzein-8C-glucoside) (Fig. 1), has become an object of interest in recent years. It is a major bioactive component found in the root of plants of the genus Pueraria (common name 
kudzu). Puerarin was isolated from such species as Pueraria lobata [10] and Pueraria mirifica [11]. Puerarin was extracted for the first time in 1950 [12]. Since then, its biological activity has been broadly analyzed. A number of health benefits are attributed to puerarin, i.e., antioxidative [13], anti-inflammatory [14], neuroprotective [15], hepatoprotective [16], anticancer [17], antidiabetic [18], cardioprotective [19], and anti-atherosclerotic effects [20]. This study reviews the current state of knowledge regarding the importance of puerarin for bone health. Based on the results of in vitro experiments and animal studies, it was discussed how it affects bone mineral density, bone markers and structural parameters.

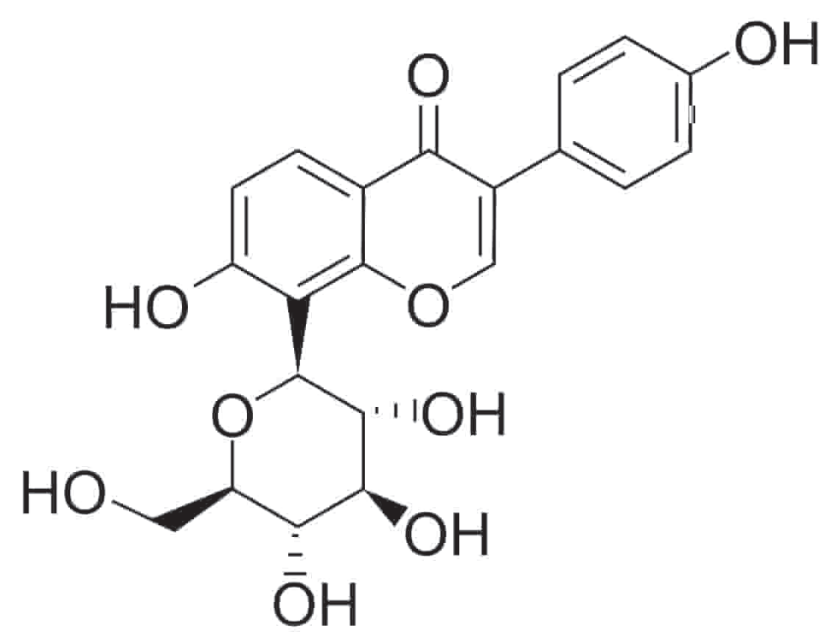

Fig. 1. Chemical structure of puerarin (daidzein-8-C-glucoside).

\section{Effect of puerarin on bone health}

\subsection{In vitro studies}

Many in vitro experiments in which the effect of puerarin on bone health is analyzed via various mechanisms, have been conducted so far (Fig. 2). In their research, scientists focused primarily on determining the effects of puerarin on osteoblasts and osteoclasts. The results obtained are discussed below.

\subsubsection{Effect on osteoblasts}

Li et al. [21] analyzed the effect of puerarin (5-20 $\mu \mathrm{M})$ on the proliferation of osteoblasts from female mice using the MTT assay. They noted that the proliferation of osteoblasts increased after 24 hours of culture. It should be noted that after $48 \mathrm{~h}$ and $72 \mathrm{~h}$ even higher proliferation was observed but this effect differed depending on the dose (5 $\mu \mathrm{M}, p<0.05 ; 10$ and $20 \mu \mathrm{M}, p<0.01)$. Additionally, the authors of the experiment found a statistically significant decrease in RANKL (receptor activator of nuclear factor kappa-B ligand) protein expression in osteoblasts after treatment with puerarin. At the same time, they noted an increase in OPG (osteoprotegerin) protein expression. These results showed that puerarin may inhibit osteoclastogenesis-related processes and thus prevent bone resorption [21]. Similar results were obtained by Yuan et al. [22] who showed that puerarin increased mRNA expression of OPG/GAPD in M3T3-E1 cells and simultaneously decreased RANKL mRNA expression. Wang et al. [23] confirmed that puerarin stimulates osteogenesis in osteoblastlike MC3T3-E1 cells. The observed increase in the viability of MC3T3-E1 cells indicates a significant effect of puerarin on proliferation, while the increase in alkaline phosphatase (ALP) activity (which is a marker of osteoblast activity) shows a stimulating effect of osteoblastic differentiation. However, the most beneficial effect was observed in the case of puerarin administration at $20 \mu \mathrm{M}$. In addition, it has been noted that puerarin may stimulate osteocalcin (a marker of bone formation) secretion in MC3T3E1 cells. The authors of the experiment suggest that puerarin may stimulate osteogenesis by up-regulating the expression of OPN (osteopontin) and OPG, which constitute bone turnover markers. The results of an experiment by Shan et al. [24] confirmed that puerarin (10-40 $\mu \mathrm{M})$ stimulates cell differentiation in MC3TC-E1 cells. As a result of treatment with puerarin, the researchers observed an increase in ALP activity, collagen type 1 secretion as well as osteocalcin secretion. Furthermore, the experiment showed an increase in bone mineralization of MC3T3-E1 cells. Reverse transcription-quantitative polymerase chain reaction (RT-qPCR) showed an increase in expression of miR-106b after treatment of MC3T3-E1 cells with puerarin. This may indicate the anti-osteoporotic properties of puerarin, as miR-106b can have an inhibitory effect on osteoclastogenesis and osteolysis. A similar effect was observed by Feng et al. [25] who confirmed that puerarin stimulates the viability and differentiation of MC3T3-E1 cells by downregulation of miR-204. An in vitro experiment published in 2012 showed that puerarin does not affect rat osteoblast proliferation and cell viability. On the other hand, it has been found that puerarin $(0.01-0.1 \mu \mathrm{M})$ may stimulate osteoblast differentiation. It was observed that cells treated with puerarin had higher ALP activity and Col I (type I collagen) secretion. In addition, this study showed that treatment with puerarin caused a significant increase in phospho-p38 MAPK (mitogen-activated protein kinase) and $\beta$-catenin proteins, indicating that puerarin could increase osteoblast differentiation via p38 MAPK and Wnt/b-catenin pathways [26]. Another experiment proved that puerarin promoted the proliferation and differentiation of human osteoblastic MG-63 cells. The highest puerarin activity was found at a dose of $1 \mu \mathrm{M}$. Additionally, it was observed that puerarin increased ALP activity as well as stimulated collagen synthesis in osteoblastic cells. It was also noted that puerarin inhibited cisplatin-induced apoptosis in MG-63 cells via ER-dependent MEK/ERK and PI3K (phosphatidylinositol 3-kinase-protein kinase B)/Akt activation [27]. Simi- 


\begin{tabular}{|c|c|}
\hline \multicolumn{2}{|c|}{ PUERARIN } \\
\hline $\begin{array}{l}\text { 个 Bone formation markers } \\
\text { and osteoblastogenesis } \\
\text { By-products of collagen synthesis } \\
\qquad \text { Collagen type I secretion } \\
\text { Osteoblast enzymes } \\
\qquad \text { Alkaline phosphatase (ALP) } \\
\text { Matrix proteins } \\
\text { • Osteocalcin (OCN) }\end{array}$ & 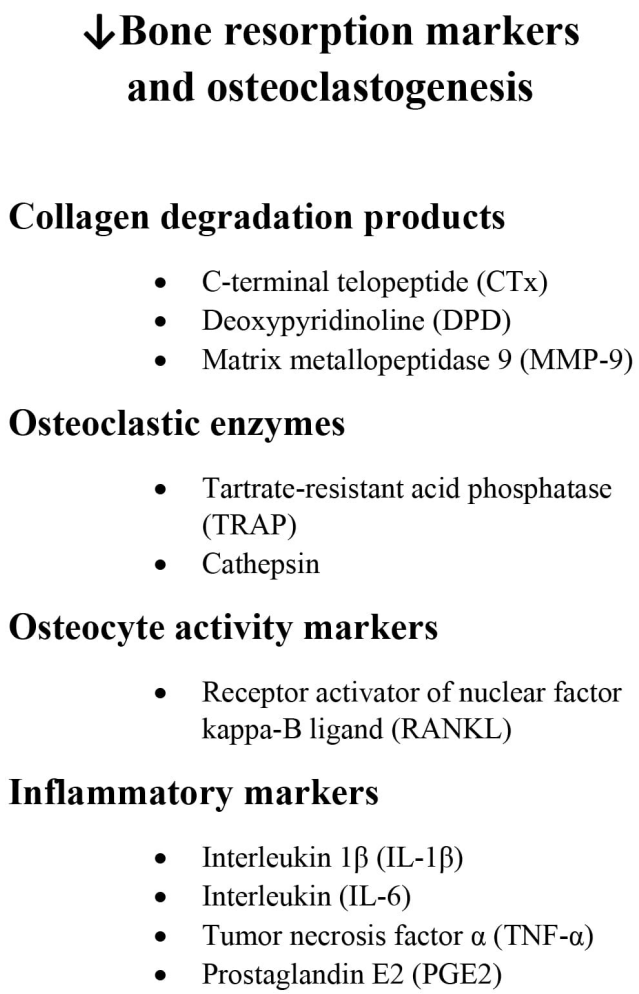 \\
\hline \multicolumn{2}{|l|}{$\begin{array}{l}\uparrow \text { Bone mineral density (BMD) } \\
\uparrow \text { Bone mineral content (BMC) } \\
\uparrow \text { Bone microarchitecture } \\
\downarrow \text { Bone resorption area / osteolysis area }\end{array}$} \\
\hline
\end{tabular}

Fig. 2. The effect of puerarin on bone health.

larly, Zhang et al. [28] concluded and proved that puerarin (2.5-100 $\mu \mathrm{M}$ ) positively affects rat calvaria osteoblastic bone formation through activation of the PI3K/AKt signaling pathway. An experiment by Liu et al. [29] has provided evidence that puerarin may prevent bone loss. The authors of the research noted that puerarin protected human osteoblasts from serum-free-induced apoptosis. The highest effectiveness was found for puerarin concentration of $10^{-8}$ M. Additionally, treatment with puerarin caused an increase in Bcl-2 (B-cell lymphoma 2) proteins while decreasing the expression of Bax in osteoblasts. It was also found that the anti-apoptotic effect was mediated by the ERK signaling pathway. Wang et al. [30] proved that puerarin (0.01-1 $\mu \mathrm{M}$ ) stimulated the production of osteoprotegerin by human osteoblastic MG-63 cells. It should be noted that the increase in OPG expression caused by puerarin was compa- rable to the effect obtained with the use of $17 \beta$-estradiol. Simultaneously, puerarin inhibits the production of RANKL and IL-6 (interleukin 6), which may indicate the inhibition of bone resorption. The researchers also showed that puerarin stimulates ER-alpha, ER-beta, and steroid hormone receptor coactivator expression, which proves that puerarin acts via the ERE (estrogen response elements) pathway. Tiyasatkulkovit et al. [31] further proved that both puerarin (1000 nM) and Pueraria mirifica (PM) extract (100 $\mu \mathrm{g} / \mathrm{mL}$ may increase the expression of ALP over type I collagen in primary baboon osteoblasts. At the same time, they did not observe the effect of puerarin and PM on other markers of bone formation: Runx2 (run-related transcription factor 2), osteocalcin and osterix. Additionally, the authors of the experiment noted that both puerarin and PM extract lowered the levels of RANKL mRNA expression (bone resorption 
marker). However, they did not cause any changes in OPG expression. An increase in ALP activity after treatment with puerarin was also observed in a study performed by Wang et al. [32] The highest ALP activity was found after the application of puerarin at $10^{-6} \mathrm{~mol} / \mathrm{L}$. At the indicated dose of puerarin, the most significant positive effect on the number of mineralized modules (5.64) was also noted. It should also be noted that too high doses of puerarin $\left(10^{-3}\right.$ $\mathrm{mol} / \mathrm{L}$ ) may inhibit ALP activity, osteoblast differentiation, reduce the formation of mineralized osteoblast nodules and, as a result, adversely affect bone formation. In turn, Wang et al. [32] proved that puerarin can stimulate proliferation and differentiation of osteoblasts in a high-glucose environment. This is an important fact because high glucose levels can disturb the balance between osteoclastic/osteogenic activity. Consistent with the obtained results, scientists suggest that puerarin may be a promising agent in the treatment of diabetic osteoporosis (DOP) [33].

\subsubsection{Effect on osteoclasts}

An experiment conducted by Yuan et al. [22] has proven that puerarin $\left(10^{-7}-10^{-6} \mathrm{~mol} / \mathrm{L}\right)$ may inhibit osteoclast formation induced by RANKL. The researchers observed that RAW264.7 cells treated with puerarin formed a lower number of TRAP-positive cells (tartrate resistant acid phosphatase). One study showed that puerarin (10$50 \mu \mathrm{M}$ ) inhibited LPS-induced (lipopolysaccharide) differentiation from osteoclast precursor RAW264.7 cells. According to the researchers, puerarin was significantly capable of inhibiting the production of pro-inflammatory cytokines: PGE2 (prostaglandin E2), IL-1 $\beta$ (interleukin $1 \beta$ ) and TNF- $\alpha$ (tumor necrosis factor alpha), which may stimulate osteoclast differentiation. In consequence, a statistically significant decrease in mRNA expression of TRAP, cathepsin K and MMP-9 (metalloproteinase 9) occurred. At the same time, it was observed that puerarin inhibited the activation of Akt in RAW264.7 cells, thus preventing osteoclastogenesis [34]. The studies conducted by Yang et al. [35] provided conclusions about the beneficial effect of puerarin $(1-25 \mu \mathrm{M})$ on the inhibition of RANKL-induced osteoclast activation in bone marrow-derived macrophages. The researchers observed that the cells treated with puerarin formed a lower number of TRAP-positive cells. Additionally, it was observed that puerarin decreased the expression of osteoclast-related genes including cathepsin $\mathrm{K}$, a nuclear factor of activated $\mathrm{T}$ cell cytoplasmic 1 and calcitonin receptor. The observed effects differed depending on the dose, and the most beneficial results were obtained after treatment with puerarin at a dose of $25 \mu \mathrm{M}$. Based on these data, puerarin was found to be able to inhibit osteoclast formation [35]. The effect of puerarin on the inhibition of osteoclastogenesis was also confirmed by Lin et al. [36]. They observed that puerarin caused the inhibition of migration of osteoclast precursors (OCP) by blocking the production of monocyte chemotactic protein-1 (MCP-1). It should be mentioned here that MCP-1 is a key mediator of osteoclastogenesis and plays a role in bone resorption.

\subsubsection{Effect on other cells}

$\mathrm{Li}$ and Peng proved that puerarin $(10-100 \mu \mathrm{M})$ stimulated the proliferation of human periodontal ligament stem cells. This effect differed depending on the dose and the increase in puerarin concentration $(100 \mu \mathrm{M})$ was correlated with greater proliferation. Additionally, they observed that puerarin contributed to ALP activity. This research also showed that puerarin increased the osteogenic differentiation of periodontal ligament stem cells [37]. Wang et al. [38] noted that the osteocalcin level and ALP activity in bone marrow cells treated with ethanol + puerarin $(0.01 \mathrm{mg} / \mathrm{mL})$ were higher compared to cells treated with ethanol only. The results obtained show that puerarin may protect bone marrow stromal cells from alcohol-induced osteonecrosis [38]. The essence of the presented results of in vitro studies on puerarin is presented in Table 1 (Ref. [2138]).

\subsection{Animal model studies}

The results obtained from in vitro studies are widely confirmed in tests carried out on animal models. It has been proven many times that the administration of puerarin had a beneficial effect on bone markers, bone mineral density and bone structural parameters.

\subsubsection{Effect on bone markers}

Li et al. [21] proved the anti-osteoporotic activity of puerarin 6-O-xyloside. They showed that ovariectomized rats $(\mathrm{OVX})$ treated with puerarin (intraperitoneal injection) (20, 40, $60 \mathrm{mg} / \mathrm{kg} / \mathrm{d})$ had significantly higher blood calcium levels compared to the control OVX group. Additionally, higher blood phosphorus levels were observed in animals treated with puerarin. However, this effect was differed depending on the dose. The highest level of the indicated components was found with the administration of puerarin at $60 \mathrm{mg} / \mathrm{kg} / \mathrm{d}$. This research also assessed ALP activity. It was observed that the use of puerarin at doses of 40 or $60 \mathrm{mg} / \mathrm{kg} / \mathrm{d}$ increased serum levels of ALP. At the same time, an increase in osteoprotegerin levels was observed [21]. Another study that proved the anti-osteoporotic effect of puerarin was conducted by Michihara et al. [39]. They showed that in ovariectomized mice during 8-week oral (per os) administration of puerarin $(5 \mathrm{mg} / \mathrm{kg} / \mathrm{d}$ ) urinary DPD (doxypyridinoline) levels were lower compared to mice that did not consume this isoflavonoid. In the conducted experiment it was observed that the control group had a significantly lower number of femoral trabeculae than the OVX-puerarin group, which indicates that puerarin may protect the trabecular structure from destruction. In the group fed with puerarin a significantly lower activity of TRAP, which is a bone absorption marker, was also found. It should be noted, 
Table 1. Effect of puerarin on biomarkers of bone health (in vitro).

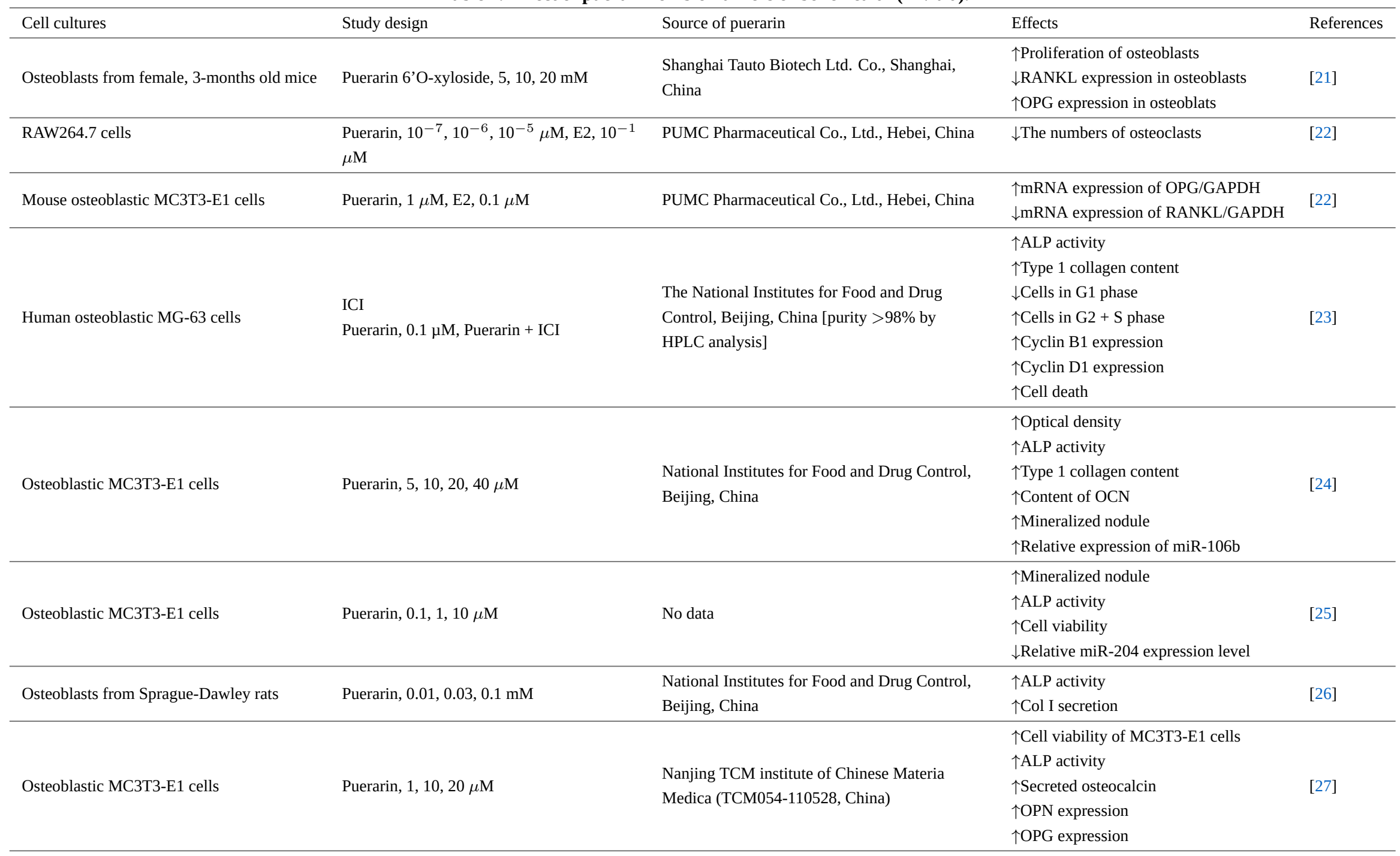


Table 1. Continued.

\begin{tabular}{|c|c|c|c|c|}
\hline Cell cultures & Study design & Source of puerarin & Effects & References \\
\hline Rat calvaria osteoblasts & $\begin{array}{l}\text { 17-beta-estriadol group, Puerarin, 2.5, 5, } \\
10,25,50,100 \mu \mathrm{mol} / \mathrm{L}\end{array}$ & $\begin{array}{l}\text { Beijing Institutes for Food and Drug Control, } \\
\text { Beijing China [purity 98.5\%] }\end{array}$ & $\begin{array}{l}\uparrow \text { ALP activity } \\
\uparrow \text { Cell viability } \\
\uparrow \text { Mineral nodules formation } \\
\uparrow \mathrm{p} \text {-Akt/Total Akt }\end{array}$ & [28] \\
\hline Human osteoblasts from femur & $\begin{array}{l}\text { Puerarin, } 10^{-10}, 10^{-9}, 10^{-8}, 10^{-7}, 10^{-6} \\
\mu \mathrm{M} \text {. } \\
\mathrm{E} 2,10 \mu \mathrm{M}\end{array}$ & Sigma, Inc., USA & $\begin{array}{l}\downarrow \text { Cell apoptosis } \\
\downarrow \text { Bax expression } \\
\uparrow \text { Bcl-2 expression }\end{array}$ & [29] \\
\hline Human osteoblastic MG-63 cells & $\begin{array}{l}\text { ICI, } 100 \mu \mathrm{M} \text {, Puerarin, } 0.1 \mu \mathrm{M} \text {, Puerarin + ICI, } \\
\text { Puerarin, } 0.01,0.1,1 \mu \mathrm{M}\end{array}$ & $\begin{array}{l}\text { National Institutes for Food and Drug Control, } \\
\text { Beijing, China [purity }>98 \% \text { ] }\end{array}$ & $\begin{array}{l}\downarrow \mathrm{IL}-6 \text { production } \\
\uparrow \mathrm{OPG} \text { expression } \\
\downarrow \mathrm{RANKL} \text { expression } \\
\uparrow \mathrm{ER} \alpha \text { expression } \\
\uparrow \mathrm{ER} \beta \text { expression }\end{array}$ & [30] \\
\hline Primary baboon osteoblasts & $\begin{array}{l}\text { P. mirifica extract, } 100 \mu \mathrm{g} / \mathrm{mL} \text {, Genistein, } \\
1000 \mathrm{nM} \text {, Puerarin, Puerarin, } 1000 \mathrm{nM}\end{array}$ & $\begin{array}{l}\text { P. mirifica (Chiang Mai Province, Northern Thai- } \\
\text { land) } \\
\text { Puerarin (LKT Laboratories Inc., St. Paul, MN, } \\
\text { USA) }\end{array}$ & $\begin{array}{l}\uparrow \text { ALP activity } \\
\uparrow \text { Type I collagen } \\
\downarrow \text { RANKL mRNA expression }\end{array}$ & [31] \\
\hline Osteoblasts from calvarial bone of Wistar rats & $\begin{array}{l}\text { Puerarin, } 10^{-10}, 10^{-9}, 10^{-8}, 10^{-7}, 10^{-6} \text {, } \\
10^{-5}, 10^{-4}, 10^{-3} \mu \mathrm{M}\end{array}$ & $\begin{array}{l}\text { National Institutes for Food and Drug Control, } \\
\text { Beijing, China }\end{array}$ & $\begin{array}{l}\uparrow \text { Osteoblast proliferation } \\
\uparrow A L P \text { activity } \\
\uparrow \text { Number of mineralized nodules }\end{array}$ & [32] \\
\hline $\begin{array}{l}\text { Osteoblasts from newly born Sprague Daw- } \\
\text { ley rats }\end{array}$ & Puerarin, $0.01,0.1,1 \mu \mathrm{M}$ & $\begin{array}{l}\text { National Institutes for Food and Drug Control } \\
\text { (NIFDC), China }\end{array}$ & $\uparrow A L P$ activity & [33] \\
\hline RAW264.7 cells & LPS + Puerarin, 10, 25, $50 \mu \mathrm{M}$ & $\begin{array}{l}\text { National Institutes for Food and Drug Control, } \\
\text { Beijing, China [purity } \geq 99.8 \% \text { by HPLC] }\end{array}$ & $\begin{array}{l}\downarrow \text { TNF- } \alpha \\
\downarrow \text { IL-1 } \beta \\
\downarrow \text { PGE2 } \\
\downarrow \text { TRAP mRNA expression } \\
\downarrow \text { Cathepsin K mRNA expression } \\
\downarrow \text { MMP-9 mRNA expression } \\
\downarrow \text { Activation of Akt }\end{array}$ & [34] \\
\hline
\end{tabular}


Table 1. Continued.

\begin{tabular}{|c|c|c|c|c|}
\hline Cell cultures & Study design & Source of puerarin & Effects & References \\
\hline $\begin{array}{l}\text { Bone marrow-derived macrophages from } \\
\text { femur of male C57BL/6J mice }\end{array}$ & Puerarin, 1, 5, $25 \mu \mathrm{M}$ & Sigma-Aldrich (St. Louis, USA) [purity $\geq 98.0 \%$ ] & $\begin{array}{l}\downarrow \text { Number of osteoclasts per wall } \\
\downarrow \text { Percentage of osteoclast area } \\
\downarrow \text { Ring number per wall } \\
\downarrow \text { Area of bone resorption } \\
\downarrow \text { CTR mRNA expression } \\
\downarrow \text { CTR mRNA expression } \\
\downarrow \text { NFATc1 mRNA expression } \\
\downarrow \text { C-fos mRNA expression }\end{array}$ & [35] \\
\hline RAW264.7 cells & Puerarin, 10, 25, 50, $100 \mu \mathrm{M}$ & No data & $\downarrow \mathrm{MCP}-1$ secretion & [36] \\
\hline $\begin{array}{l}\text { Human periodontal ligament stem cells } \\
\text { (PDLSC) }\end{array}$ & Puerarin, 10, 50, $100 \mu \mathrm{M}$ & Inchem Corp., Rock Hill, SC, USA [purity $\geq 95 \%$ ] & $\begin{array}{l}\uparrow \mathrm{MTT} \text { optical density } \\
\uparrow \mathrm{ALP} \text { activity } \\
\uparrow \mathrm{mRNA} \text { expression of RUNX2 } \\
\uparrow \mathrm{mRNA} \text { expression of collagen I } \\
\uparrow \mathrm{mRNA} \text { expression of OPN } \\
\uparrow \mathrm{mRNA} \text { expression of OCN }\end{array}$ & [37] \\
\hline $\begin{array}{l}\text { Bone marrow cells } \\
\text { from the midshafts } \\
\text { of 6- to 8-week-old } \\
\text { male and female } \\
\text { mouse femurs }\end{array}$ & Puerarin, $0.01 \mathrm{mg} / \mathrm{mL}$ & - & $\begin{array}{l}\uparrow \text { ALP activity } \\
\uparrow \text { Osteocalcin level }\end{array}$ & [38] \\
\hline
\end{tabular}

ALP, Alkaline phosphatase; BAP, Bone alkaline phosphatase; BMC, Bone mineral content; BMD, Bone mineral density; BV/TV, Bone surface/total volume; BS, Bone surface; Ct, Cortical bone tissue; CTx, C-terminal telopeptide; Ca, calcium; DPD, Deoxypyridinoline; EE, 17 alpha-ethinylestradiol; FM, Femoral metaphysis; FD, Femoral diaphysis; L4, Fourth lumbar vertebra; LPS, Lipopolysaccharides; MCF-7 cell, epithelial luminal cell line; MMP, Matrix metalloproteinase; NFATc1, Nuclear factor of activated T-cell, cytoplasmic 1; Ob.S, Osteoblast surface; Oc.S, Osteoclast surface; OVX, ovariectomized group; ORX, orchidectomized; OPG, Osteoprotegerin; OPN, Osteopontin; PPAR $\gamma$, Peroxisome proliferator-activated receptor $\gamma$; PTIF, total isoflavones from Pueraria lobata; PVEE, kudzu vine ethanol extracts; RANKL, receptor activator of nuclear factor- $\kappa$ B ligand; Tb, Trabecula; Tb.N/Tb.Tn, Trabecular number; Tb.Sp, Trabecular separation; TD, Tibial diaphysis; TM, Tibial metaphysis; TRAP, Tartrate resistant acid phosphatase. 
however, that the administration of puerarin did not affect the level of osteocalcin. Based on the observed lack of effect of puerarin on morphology and weight of oviducts and uterus, the researchers indicated that the observed properties are independent of the estrogen receptor-mediated pathway. They also proved this effect by observing that puerarin did not stimulate the growth of MCF-7 cells [39]. Similarly, Tanaka et al. [40] noted that puerarin may inhibit ovariectomy-induced bone loss. In the experiment, the researchers administered kudzu vine ethanol extract (PVEE) per os to female mice. Of isoflavones present in it, puerarin was found in the highest amounts (52\%). This extract was administered at $20 \mathrm{mg} / \mathrm{kg} / \mathrm{d}$ for 8 weeks. The authors of this research showed that animals which consumed PVEE had lower levels of bone resorption markers such as DPD and TRAP compared to OVX mice. At the same time, it was noted that the use of PVEE inhibited the reduction in femoral bone mineral density. A lower number of matured osteoclasts in the distal femur was also observed in the group treated with PVEE. On the other hand, administration of PVEE did not affect the serum bone-specific alkaline phosphatase activity. At the same time, Liang et al. [41] noted that puerarin may have a beneficial effect on bones in rats with diabetes. It was observed that daily intraperitoneal administration of puerarin $(60 \mathrm{mg} / \mathrm{kg} / \mathrm{d})$ caused a decrease in caspase- 3 expression in osteoblasts. The authors of the experiment suggest that high blood glucose levels are associated with an increase in the expression of caspase-3, which in turn may contribute to osteoblast apoptosis and lead to the development of osteoporosis. Moreover, Luo et al. [42] proved that oral administration of kudzu root extract $(0.45,0.9$ and $1.8 \mathrm{~g} / \mathrm{kg} / \mathrm{d})$ to ovariectomized rats may have a beneficial effect on bone health. In the conducted studies it was observed that the use of kudzu root extract resulted in lowering the serum levels of C-terminal telopeptide of collagen type I (CTx). This indicates that this extract may inhibit bone resorption. Additionally, it has been shown that the administration of $P$. lobata extract $(100 \mathrm{mg} / \mathrm{kg} / \mathrm{d})$ results in a statistically significant reduction in the levels of osteocalcin as well as ALP and CTx, which are markers of bone resorption and remodeling [43]. It is also worth mentioning that studies conducted by Wang et al. [38] on an animal model showed that puerarin may inhibit alcohol-induced osteonecrosis. Marrow and bone necrosis were found in mice belonging to the model group treated with ethanol (intragastric). An increase in the number of empty osteocyte lacunae in the femur was also observed. Such adverse lesions were not found in mice treated with intramuscular injection of puerarin $(0.5 \mathrm{~g} / \mathrm{kg} / \mathrm{d})$. Additionally, higher expression of osteocalcin mRNA was found in animals treated with puerarin [38]. Research results published in 2020 also indicated that puerarin (15.4 and $30.8 \mathrm{mg} / \mathrm{kg} / \mathrm{d}$ ) administered i.p. inhibits titanium particleinduced osteolysis [44]. Zhang et al. [34], on the other hand, proved that calvarial injection of puerarin $(1 \mathrm{mg} / \mathrm{kg} / \mathrm{d})$ is capable of inhibiting LPS-induced bone loss. The authors found that after the injection of LPS there was a significant increase in the number of osteoclasts and an increase in the area of osteolysis. At the same time, significant reductions in calvaria weight were observed. Mice additionally treated with puerarin had a significantly lower number of osteoclasts as well as decreased osteolysis area. Lower bone mass loss, compared to the puerarin-free group (68.5 vs. $47.9 \mathrm{mg}$ of calvaria weight), was observed as well [45]. The beneficial effect of Pueraria lobata extract (PE) was also confirmed by Lee et al. [46]. They observed that the administration of PE (25-1600 mg/kg) to ovariectomized (OVX) rats resulted in a reduction in the level of bone turnover markers, such as osteocalcin, C-terminal telopeptide fragment of type I collagen, deoxypyridinoline, and pyridinoline, the concentration of which was elevated in OVX rats. The highest effect was seen in animals receiving $1600 \mathrm{mg}$ PE $/ \mathrm{kg}$. Moreover, researchers confirmed that puerarin caused an increase in the plasma level of estradiol, which indicates that PE has estrogenic activity and its activity is similar to that of phytoestrogens. Therefore, it seems that puerarin may slow down the osteoporotic changes associated with estrogen deficiency, which is characteristic in postmenopausal women [46].

\subsubsection{Effect on bone mass/bone mineral density}

An experiment by Tanaka et al. [40] showed that ovariectomized mice fed with kudzu vine ethanol extracts (PVEE) had a lower degree of trabecular destruction than mice belonging to the control OVX-group. The beneficial effect of puerarin on bones was also noted in a research conducted by Yuan et al. [22]. Ovariectomized mice received a diet containing puerarin at different doses: 2,4 or $8 \mathrm{mg} / \mathrm{d}$. The authors observed that mice receiving food enriched with puerarin $(2 \mathrm{mg} / \mathrm{d})$ had a higher mineral density of various regions of the femur - proximal femur, middle femur, distal femur compared to animals fed without this additive [22]. In an experiment conducted by Liang et al. [41], femur X-rays showed that animals treated with puerarin had a lower loss of bone mass compared to those not treated with this additive. It was shown that femoral BMD (bone mineral density) in rats treated with puerarin was $9.7 \%$ higher than in the control group. Moreover, rats with diabetes had a higher number of osteoclasts clumped together as well as higher cortical bone reduction. In rats treated with puerarin, these lesions were milder [41]. Similar results were also obtained by Cho et al. [47] who administered isoflavones from Pueraria lobata per os to ovariectomized mice. Of isoflavones found in P. lobata extract, puerarin was present at the highest concentration (7.5\%). Daidzein (4.2\%) and genistein $(1.9 \%)$ were present in smaller amounts. The researchers found that mice fed with P. lobata extract (200 and $500 \mathrm{mg} / \mathrm{kg} / \mathrm{d}$ ) had significantly higher femoral BMD than those fed without this additive [47]. Similarly, in another study, researchers showed that rats consuming the 
kudzu root had higher BMD of the middle femur [42]. In turn, Wang et al. [26] proved that puerarin stimulates bone formation. They showed that ovariectomized rats treated with puerarin (i.g.; $20 \mathrm{mg} / \mathrm{kg} / \mathrm{d}$ ) had a higher femur BMD and BMC (bone mineral content). The positive effect of Pueraria mirifica administration on bone health was also noted by Suthon et al. [48]. The researchers showed that rats treated with $\mathrm{P}$. mirifica $(50 \mathrm{mg} / \mathrm{kg} / \mathrm{d}$ ) by gavage had a higher total BMD of L4 (fourth lumbar vertebra), as well as trabecular BMD of L4 and tibial metaphysis, compared to rats not treated with isoflavones. The effect of dietary isoflavones from Puerariae Radix on bone metabolism in ovariectomized rats was also studied by Lim et al. [43]. The researchers administered orally $P$. lobata extract, containing high amounts of isoflavones, to rats. Puerarin constituted $57.6 \%$ of all isoflavones. The remaining isoflavones were daidzein (30.4\%) and genistein (12.0\%). They found that rats treated with the extract at $100 \mathrm{mg} / \mathrm{kg} / \mathrm{d}$ had a significantly higher femoral bone mineral density compared to the control OVX-group. Such an effect was not observed in the group treated with $P$. lobata extract at $30 \mathrm{mg} / \mathrm{kg} / \mathrm{d}$. Yang et al. [49] showed that rats with ligature-induced periodontitis and treated with puerarin at $200 \mathrm{mg} / \mathrm{kg} / \mathrm{d}$ by gavage had a lower volume of bone loss compared to those not treated with isoflavonoids. In this research, it was noted that the consumption of puerarin inhibited alveolar bone loss by inhibiting RANKL production and reducing the number of active osteoclasts [49]. The experiment performed on healthy male C57BL/6J mice showed that the intraperitoneal administration of puerarin (10 and $50 \mathrm{mg} / \mathrm{kg} / \mathrm{d}$ ) caused inhibition of osteolysis induced by titanium particles. The authors of the research noted that mice treated with puerarin had higher BMD and BV/TV (bone surface/total volume). However, this effect was dose-dependent, and a more beneficial effect was observed when a higher dose of puerarin was used. In addition, micro-CT scanning showed a lower number of pores in mice treated with puerarin. The researchers also noted a statistically significant reduction in eroded surface area, in the number of TRAP-positive cells, as well as in the osteoclast surface to bone surface ratio [49]. Interesting research results were observed by Liu et al. [50], who noted that administration of puerarin combined with zinc (50 mg/kg/d + $0.25 \mathrm{mg} / \mathrm{kg} / \mathrm{d}$ ) by gavage could prevent mandibular bone loss in OVX rats more effectively than administration of these compounds separately. The experiment showed that rats treated with puerarin + zinc had statistically significantly higher bone mineral density compared to rats fed without these additives, as well as compared to those consuming puerarin + zinc separately. In contrast, studies performed on adult ovariectomized rats showed that rats treated with puerarin (administered i.p.) (50 mg/kg/d) from Pueraria lobata had a lower BMD of the proximal tibia compared to animals not treated with this isoflavonoid. This means that puerarin was not capable of reducing loss of BMD induced by ovariectomy
[47]. In 2020, the results of a meta-analysis on the effect of puerarin administration on bone mass for OVX-induced postmenopausal osteoporosis in a murine model were published. Based on eight randomized studies, the authors showed that the use of puerarin can have a beneficial effect on the increase in bone mineral density [51]. The beneficial effect of puerarin on bones was also noted by Li et al. [52]. They showed that administration of puerarin at 100 $\mathrm{mg} / \mathrm{kg} / \mathrm{d}$ resulted in a statistically significant increase in cortex and trabeculae BMD. Simultaneously, the authors of the experiment noticed that in the tested rats there was an improvement in the integrity of the intestinal mucosa. Moreover, positive changes in gut microbiota were noted. For example, Lactobacillaceae and Bifidobacteriaceae bacteria were found to grow. Additionally, puerarin increased the content of SCFAs. The authors suggested that puerarin may prevent osteoporotic changes by modulating the community of gut microbiota and repairing intestinal mucosal integrity [52]. The essence of the presented results of puerarin studies in animal models is presented in Table 2 (Ref. [21, 22, 26, 34, 35, 38-50, 52-56]).

\subsubsection{Effect on structural parameters (histomorphometric data)}

Li et al. [21] observed that the administration of puerarin 6-O-xyloside to rats had a beneficial effect on bone microarchitecture. Compared to the control OVX group, in animals treated with puerarin, the cortical and trabecular bone were thickened. It should be noted that the level of pathological lesions in femoral bone tissue was dependent on the dose of puerarin administered. The least adverse lesions occurred after administration of $60 \mathrm{mg}$ puerarin $/ \mathrm{kg} / \mathrm{d}$ [21]. Urasopon et al. [53] conducted research in which they administered Pueraria mirifica to rats after orchidectomy. They found that the enrichment of food with P. mirifica inhibited bone loss in trabecular and cortical bones. It should be stressed that this effect was dependent on the dose of $P$. mirifica. The most positive results were achieved in the group of rats treated with the highest amounts of $P$. mirifica $(1000 \mathrm{mg} / \mathrm{kg} / \mathrm{d})$ [53]. Wang et al. [26] showed that the administration of puerarin to rats resulted in the improvement of trabecular bone structure. They noted that both trabecular number and trabecular thickness were significantly higher in the OVX + puerarin group compared to the OVX group [26]. The beneficial effect on bone histomorphometry was also confirmed by Suthon et al. [48]. They observed that rats treated with $P$. mirifica showed an improvement in such parameters as bone volume (BV), trabecular separation (Tb.Sp), trabecular number (Tb.N), and osteoblast surface (Ob.S) [48]. Similarly, Lim et al. [43] observed that puerarin can effectively improve the histomorphometry parameters. It was noted that the proximal tibia had significantly higher trabecular number (Tb.N) as well as BV/TV. However, no difference was found for such parameters as bone surface/bone volume (BS/BV), trabecular thickness 
Table 2. Effect of puerarin on biomarkers of bone health in animal models.

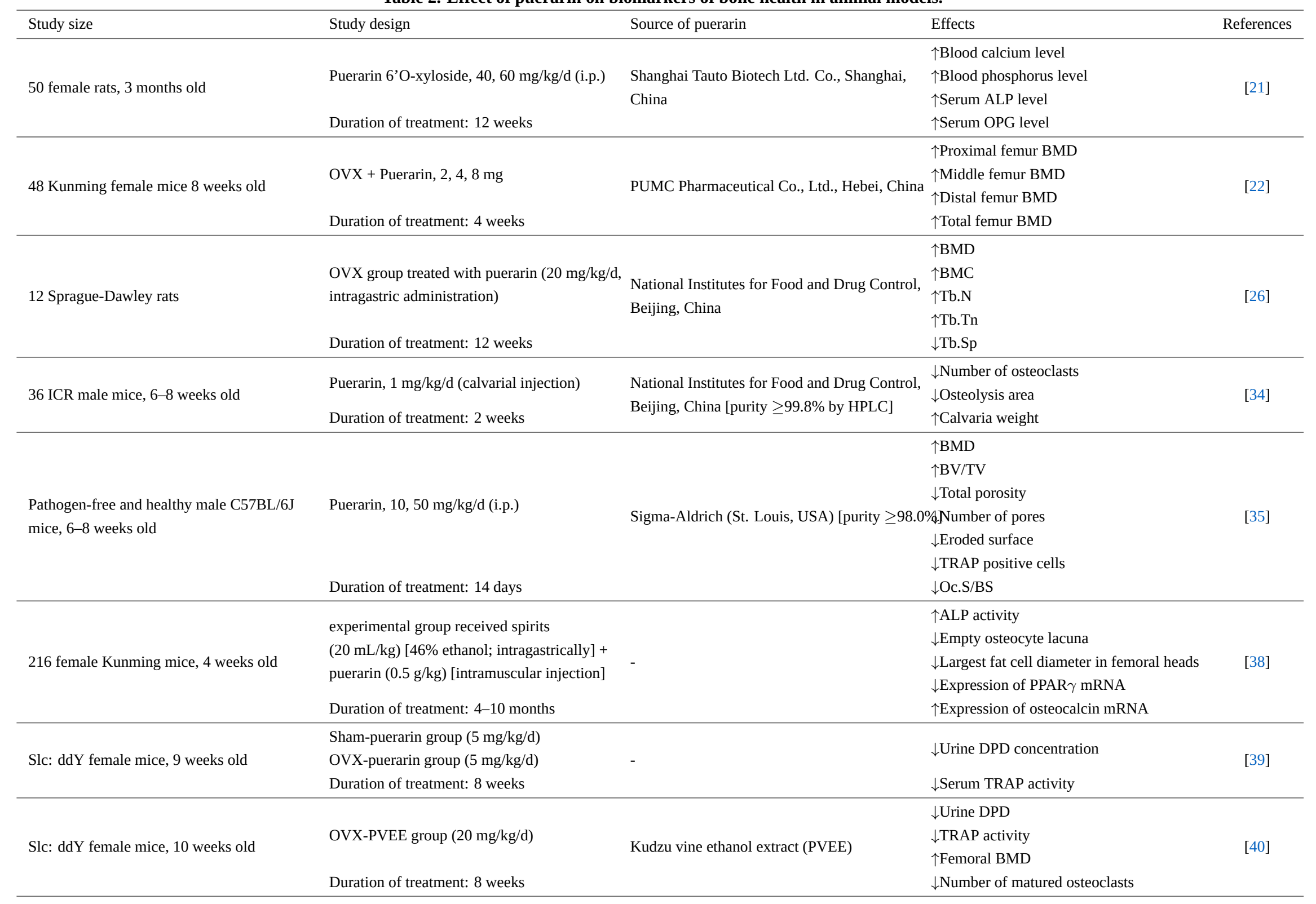


Table 2. Continued.

\begin{tabular}{|c|c|c|c|c|}
\hline Study size & Study design & Source of puerarin & Effects & References \\
\hline 30 Sprague-Dawley male rats & $\begin{array}{l}\text { Diabetes treated with puerarin } \\
\text { (intraperitoneally injected, } 60 \mathrm{mg} / \mathrm{kg} / \mathrm{d} \text { ) } \\
\text { control group } \\
\text { Duration of treatment: } 6 \text { weeks }\end{array}$ & $\begin{array}{l}\text { Limin Pharmaceutical Corporation, Jinan, } \\
\text { Shandong, China }\end{array}$ & $\begin{array}{l}\downarrow \text { Caspase-3 expression } \\
\uparrow \text { Femoral BMD) } \\
\uparrow \text { Number of osteoclasts clumped together } \\
\downarrow \text { Cortical bone reduction } \\
\uparrow \text { Deteriorated bone micro-architecture }\end{array}$ & [41] \\
\hline 60 female Sprague-Dawley rats, 6 months old & $\begin{array}{l}\text { OVX + kudzu, } 0.45,0.9,1.8 \mathrm{~g} / \mathrm{kg} / \mathrm{d} \\
\text { Duration of treatment: } 6 \text { weeks }\end{array}$ & Kudu root extract & $\begin{array}{l}\downarrow \text { Serum CTX-I } \\
\uparrow \text { Femoral BMD }\end{array}$ & [42] \\
\hline Female Sprague-Dawley rats, 8 weeks old & $\begin{array}{l}\text { OVX }+17 \beta \text {-estradiol }(10 \mu \mathrm{g} / \mathrm{kg} / \mathrm{d} \text {, i.p.) } \\
\text { OVX + PTIF ( } 30 \text { or } 100 \mathrm{mg} / \mathrm{kg} / \mathrm{d} \text { ) } \\
\text { Duration of treatment: } 8 \text { weeks }\end{array}$ & P. lobata extract (Kapdang Co., Seoul, Korea) & $\begin{array}{l}\downarrow \text { Femur BMD } \\
\downarrow \text { ALP } \\
\downarrow \text { Osteocalcin } \\
\downarrow \text { CTx } \\
\uparrow \text { Estradiol }\end{array}$ & [43] \\
\hline 20 Male Sprague-Dawley rats & Puerarin, 15.4, $30.8 \mathrm{mg} / \mathrm{kg} / \mathrm{d}$ (i.p.) & Sigma-Aldrich, Saint Louis, MO, USA & $\begin{array}{l}\uparrow \mathrm{BMD} \\
\uparrow \mathrm{BV} / \mathrm{TV} \\
\uparrow \mathrm{Tb} . \mathrm{Th} \\
\downarrow \mathrm{NFAT}-\mathrm{c1} \text { positive cells } \\
\downarrow \mathrm{MMP9-positive} \mathrm{cells}\end{array}$ & [44] \\
\hline 60 adult Sprague-Dawley rats & $\begin{array}{l}\text { Estradiol, } 10 \mu g / k g \text { (i.p.) } \\
\text { Puerarin, } 50 \mathrm{mg} / \mathrm{kg} \text { (i.p.) } \\
\text { Duration of treatment: } 12 \text { weeks }\end{array}$ & Beijing Four Rings Biopharmaceutical Co., Ltd. & $\begin{array}{l}\uparrow \mathrm{Tb} . \mathrm{N} \\
\uparrow \mathrm{BV} / \mathrm{TV} \\
\uparrow \mathrm{Osteocalcin} \\
\downarrow \mathrm{BMD}\end{array}$ & [45] \\
\hline 48 nine-week-old female Sprague Dawley rats & $\begin{array}{l}\text { Puerarin, 25, 100, 400, } 1600 \text { mg/kg/d } \\
\text { Duration of treatment: } 8 \text { weeks }\end{array}$ & Sejun F \& B Co., Ltd., Gangwon, Korea & $\begin{array}{l}\downarrow \text { Osteocalcin } \\
\downarrow \text { C-terminal telopeptide fragment of type I collagen } \\
\downarrow \text { Deoxypyridinoline } \\
\downarrow \text { Pyridinoline }\end{array}$ & [46] \\
\hline 20 female mice, 30 weeks old & $\begin{array}{l}\text { OVX treated with } 200 \mathrm{mg} \text { or } 500 \mathrm{IPL} / \mathrm{kg} / \mathrm{d} \\
\text { Duration of treatment: } 4 \text { weeks }\end{array}$ & Pueraria lobata (Willd.) extract & $\uparrow$ Femoral BMD & [47] \\
\hline Sprague-Dawley female rats, 6 months old & $\begin{array}{l}\text { OVX + P. mirifica, 5, 25, } 50 \mathrm{mg} / \mathrm{g} / \mathrm{d} \\
\text { (by gavage) OVX + Puerarin, } 7 \mathrm{mg} / \mathrm{kg} / \mathrm{d} \\
\text { (subcutaneously injected) } \\
\text { Duration of treatment: } 12 \text { weeks }\end{array}$ & P. mirifica powder (Smith Natural Co., Ltd) & $\begin{array}{l}\uparrow \text { Trabecular BMD of tibia metaphysis } \\
\uparrow \text { Total BMD of L4 } \\
\uparrow \text { Trabecular BMD of L4 } \\
\uparrow \text { BV/TV } \\
\downarrow \text { Tb.Sp } \\
\uparrow T b . N \\
\uparrow O b . S / B S\end{array}$ & [48] \\
\hline
\end{tabular}


Table 2. Continued.

$\begin{array}{lll}\text { Study size } & \text { Study design } & \text { Source of puerarin }\end{array}$

Effects

References

Shanghai Winherb Medical S\&T Development $\downarrow$ Number of RANKL-positive cells

Co. Ltd., Shanghai, China [98\% purity] $\quad \downarrow$ RANKL/OPG expression

$\downarrow$ Number of OPG-positive cells

$\downarrow$ Number of active osteoclasts

$\uparrow$ BMD in bone trabecula of mandible

OVX +17 beta-estradiol group $(10 \mathrm{ug} / \mathrm{kg} / \mathrm{d})$

Female Sprague-Dawley rats, 8 weeks old

Sigma-Aldrich, Saint Louis, MO,

$\uparrow \mathrm{BV} / \mathrm{TV}$

$\uparrow \mathrm{Tb} / \mathrm{Tb} . \mathrm{N}$

$\downarrow$ Tb.Sp

OVX + puerarin group $(50 \mathrm{mg} / \mathrm{kg} / \mathrm{d})$

OVX + zinc group $(0.25 \mathrm{mg} / \mathrm{kg} / \mathrm{d})$

$\mathrm{OVX}+$ puerarin + zinc group

Duration of treatment: 12 weeks

USA [Analytically pure]

$\uparrow$ Ca

$\downarrow$ TRAP

$\downarrow$ RANKL

vs. OVX+puearin or OVX+zinc group

Puerarin, $50,100 \mathrm{mg} / \mathrm{kg} / \mathrm{d} \quad$ J\&K Scientific Ltd, Beijing, $\quad \uparrow$ Cortex BMD

Duration of treatment: 14 weeks China [purity $\geq 98.0 \%$ ]

$\uparrow$ Trabeculae BMD

[52]

40 10-week-old Sprague-Dawley female rat

ORX + P. mirifica, 0, 10, 100, $1000 \mathrm{mg} / \mathrm{kg} / \mathrm{d}$

Pueraria mirifica (Chiang Mai Province,

TbBMD of TM/FM/L4

Male Sprague-Dawley rats, 7 months old

Thailand)

$\uparrow \mathrm{CtBMD}$ of TM/FM/L4/TD

$\uparrow$ TbBMC of TM/FM/L4

$\uparrow \mathrm{CtBMC}$ of TM/FM/L4/TD/FD

Duration of treatment: 3 months

$\uparrow \mathrm{BV} / \mathrm{TV}$

Puerarin, 100 mg/kg intraperitoneal injections

every two days

Sigma-Aldrich, Sydney, Australia

$\uparrow \mathrm{BS} / \mathrm{BV}$

$\uparrow \mathrm{BS} / \mathrm{TV}$

$\uparrow T b . N$.

$\uparrow \mathrm{BV} / \mathrm{TV}$

$\uparrow$ Tb.N

$\downarrow$ Tb.Sp

$\downarrow \mathrm{OPG}$

$\downarrow$ CTX

Duration of treatment: 14 weeks

BMD

Fermented puerarin, 100 mg/kg/d

$\uparrow \mathrm{BV} / \mathrm{TV}$

30 female 6-week-old outbred ICR mice

Daegu University, Daegu, Korea

$\uparrow$ Tb.Th

$\uparrow$ Tb.N

$\downarrow \mathrm{Tb} . \mathrm{Sp}$

Duration of treatment: 12 weeks

ALP, Alkaline phosphatase; BAP, Bone alkaline phosphatase; BMC, Bone mineral content; BMD, Bone mineral density; BV/TV, Bone surface/total volume; BS, Bone surface; Ct, Cortical bone tissue; CTx, C-terminal telopeptide; Ca, calcium; DPD, Deoxypyridinoline; EE, 17 alpha-ethinylestradiol; FM, Femoral metaphysis; FD, Femoral diaphysis; L4, Fourth lumbar vertebra; LPS, Lipopolysaccharides; MCF-7 cell, epithelia luminal cell line; MMP, Matrix metalloproteinase; NFATc1, Nuclear factor of activated T-cell, cytoplasmic 1; Ob.S, Osteoblast surface; Oc.S, Osteoclast surface; OVX, ovariectomized group; ORX, orchidectomized; OPG, Osteoprotegerin; OPN, Osteopontin; PPAR $\gamma$, Peroxisome proliferator-activated receptor $\gamma$; PTIF, total isoflavones from Pueraria lobata; PVEE, kudzu vine ethanol extracts; RANKL, receptor activator of nuclear factor- $\kappa$ B ligand; Tb, Trabecula; Tb.N/Tb.Tn, Trabecular number; Tb.Sp, Trabecular separation; TD, Tibial diaphysis; TM, Tibial metaphysis; TRAP, Tartrate resistant acid phosphatase. 
(Tb.Th) and Tb.Sp. This research also showed an increase in plasma osteocalcin level in the group treated with puerarin, with a simultaneous slight decrease in alkaline phosphatase activity [43]. Additionally, the bone morphometry of the trabeculae of mandibles proved that animals treated with puerarin + zinc showed higher values for such parameters as $\mathrm{BV} / \mathrm{TV}$ and $\mathrm{Tb} / \mathrm{Tb}$.N. At the same time, a significant reduction in Tb.Sp compared to OVX + puerarin and OVX + zinc group was noted. The authors also compared the levels of serum bone biochemical markers. They showed significantly higher calcium levels and simultaneously lower levels of TRAP and RANKL. The results of this study showed that the combined use of puerarin and zinc may be an effective way to inhibit osteoclastogenesis [50]. Xiao et al. [54] confirmed that puerarin protects against bone mass loss in ovariectomy-induced osteoporosis model mice. They reported that the animals that received puerarin had improved the bone structural features. The following parameters were improved: BV/TV, BS/BV, BS/TV, Tb.N. The authors of the experiment proved that puerarin has a bone protective effect by suppressing osteoclastogenesis via inhibition of the TRAF6/ROS-dependent MAPK/NF- $\kappa$ B signaling pathway [54]. Interesting observations were made by Guo et al. [55], who proved that puerarin inhibits the osteoporotic changes associated with streptozotocin (STZ)-induced diabetes. The authors observed that puerarin improves bone microstructure. Higher $\mathrm{BV} / \mathrm{TV}$ and Tb.N values were noted in rats receiving puerarin. At the same time, the reduction in Tb.Sp was noted. Moreover, lower levels of OPG and CTX were found. The scientists indicated that this effect may be related to suppressed inflammation [55]. Anti-osteoporotic activity was also demonstrated by Pueraria lobata fermented (FPE) with Lactobacillus paracasei JS1. Researchers observed that ovariectomized mice treated with FPE were characterized by improved bone architecture. BV/TV, Tb.Th, and Tb.N were higher compared to OVX group. At the same time, a lower value of Tb.Sp was noted. Additionally, an increase in bone mineral density was noted in mice receiving FBE [56].

\section{Summary and perspective}

The current state of knowledge clearly shows that puerarin has a positive effect on bone health. It has been repeatedly proven that this compound stimulates osteoblast differentiation and simultaneously inhibits osteoclastogenesis. The studies carried out on an animal model proved that puerarin has anti-osteoporotic properties and can inhibit bone loss in ovariectomized and orchidectomized animals. Many experiments showed that animals treated with puerarin had higher bone mineral density and bone mineral content. It should be noted, however, that despite promising indications concerning the beneficial effect of puerarin on bones, there is a lack of research that would prove such an effect among people. For this reason, the initiation of intervention studies with the participation of people, especially because there are good grounds for this, is worth considering.

\section{Author contributions}

Conceptualization and methodology—BK, AGM; investigation and data curation-BK, AS; writingoriginal draft preparation-BK; writing-review and editing-BK, JS, AGM; supervision-AGM.

\section{Ethics approval and consent to participate}

Not applicable.

\section{Acknowledgment}

Thanks to all the peer reviewers for their opinions and suggestions.

\section{Funding}

This research received no external funding.

\section{Conflict of interest}

The authors declare no conflict of interest.

\section{References}

[1] Pouresmaeili F, Kamalidehghan B, Kamarehei M, Goh MG. A comprehensive overview on osteoporosis and its risk factors. Therapeutics and Clinical Risk Management. 2018; 14: 2029_ 2049.

[2] Chen L, Ko N, Chen K. Medical Treatment for Osteoporosis: from Molecular to Clinical Opinions. International Journal of Molecular Sciences. 2019; 20: 2213.

[3] Brzezińska O, Łukasik Z, Makowska J, Walczak K. Role of vitamin $\mathrm{C}$ in osteoporosis development and treatment - a literature review. Nutrients. 2020; 12: 2394.

[4] des Bordes J, Prasad S, Pratt G, Suarez-Almazor ME, LopezOlivo MA. Knowledge, beliefs, and concerns about bone health from a systematic review and metasynthesis of qualitative studies. Public Library of Science One. 2020; 15: e0227765.

[5] Yao P, Bennett D, Mafham M, Lin X, Chen Z, Armitage J, et al. Vitamin D and Calcium for the Prevention of Fracture. JAMA Network Open. 2019; 2: e1917789.

[6] Aghajanian P, Hall S, Wongworawat MD, Mohan S. The Roles and Mechanisms of Actions of Vitamin C in Bone: New Developments. Journal of Bone and Mineral Research. 2015; 30: 1945-1955.

[7] Sahni S, Mangano KM, McLean RR, Hannan MT, Kiel DP. Dietary Approaches for Bone Health: Lessons from the Framingham Osteoporosis Study. Current Osteoporosis Reports. 2015; 13: 245-255.

[8] Sacco SM, Horcajada M, Offord E. Phytonutrients for bone health during ageing. British Journal of Clinical Pharmacology. 2013; 75: 697-707. 
[9] Zheng X, Lee S, Chun OK. Soy Isoflavones and Osteoporotic Bone Loss: a Review with an Emphasis on Modulation of Bone Remodeling. Journal of Medicinal Food. 2016; 19: 1-14.

[10] Zhou Y, Zhang H, Peng C. Puerarin: a review of pharmacological effects. Phytotherapy Research. 2014; 28: 961-975.

[11] Kakehashi A, Yoshida M, Tago Y, Ishii N, Okuno T, Gi M, et al. Pueraria mirifica Exerts Estrogenic Effects in the Mammary Gland and Uterus and Promotes Mammary Carcinogenesis in Donryu Rats. Toxins. 2016; 8: 275

[12] Wei S, Chen Y, Xu X. Progress on the pharmacological research of puerarin: a review. Chinese Journal of Natural Medicines. 2014; 12: 407-414

[13] Xiao C, Li J, Dong X, He X, Niu X, Liu C, et al. Anti-oxidative and TNF- $\alpha$ suppressive activities of puerarin derivative (4AC) in RAW264.7 cells and collagen-induced arthritic rats. European Journal of Pharmacology. 2011; 666: 242-250.

[14] Jeon Y, Lee J, Lee Y, Kim D. Puerarin inhibits inflammation and oxidative stress in dextran sulfate sodium-induced colitis mice model. Biomedicine \& Pharmacotherapy. 2020; 124: 109847.

[15] Zhu G, Wang X, Wu S, Li X, Li Q. Neuroprotective effects of puerarin on 1-methyl-4-phenyl-1,2,3,6-tetrahydropyridine induced Parkinson's disease model in mice. Phytotherapy Research. 2014; 28: 179-186.

[16] Zhao M, Du Y, Yuan L, Wang N. Protective effect of puerarin on acute alcoholic liver injury. The American Journal of Chinese Medicine. 2010; 38: 241-249.

[17] Ahmad B, Khan S, Liu Y, Xue M, Nabi G. Molecular mechanisms of anticancer activities of puerarin. Cancer Management and Research. 2020; 12: 79-90.

[18] Chen X, Yu J, Shi J. Management of Diabetes Mellitus with Puerarin, a Natural Isoflavone from Pueraria lobata. The American Journal of Chinese Medicine. 2018; 46: 1771-1789.

[19] Li X, Yuan T, Chen D, Chen Y, Sun S, Wang D, et al. Cardioprotective effects of puerarin-V on isoproterenol-induced myocardial infarction mice is associated with regulation of PPARY/NF-kB pathway. Molecules. 2018; 23: 3322.

[20] Bao L, Zhang Y, Wei G, Wang Y, Ma R, Cheng R, et al. The antiatherosclerotic effects of puerarin on induced-atherosclerosis in rabbits. Biomedical Papers of the Medical Faculty of the University Palacky Olomouc Czechoslovakia. 2015; 159: 53-59.

[21] Li H, Chen B, Pang G, Chen J, Xie J, Huang H. Anti-osteoporotic activity of puerarin 6"-O-xyloside on ovariectomized mice and its potential mechanism. Pharmaceutical Biology. 2016; 54: 111-117.

[22] Yuan S, Sheng T, Liu L, Zhang Y, Liu X, Ma T, et al. Puerarin prevents bone loss in ovariectomized mice and inhibits osteoclast formation in vitro. Chinese Journal of Natural Medicines. 2016; 14: 265-269.

[23] Wang N, Wang X, Cheng W, Cao H, Zhang P, Qin L. Puerarin promotes osteogenesis and inhibits adipogenesis in vitro. Chinese Medicine. 2013; 8: 17.

[24] Shan Z, Cheng N, Huang R, Zhao B, Zhou Y. Puerarin promotes the proliferation and differentiation of MC3T3-E1 cells via microRNA-106b by targeting receptor activator of nuclear factor-кB ligand. Experimental and Therapeutic Medicine. 2018; 15: 55-60.

[25] Feng Q, Cheng SY, Yang R, Zeng XW, Zhao FM, Zhan XQ. Puerarin promotes the viability and differentiation of MC3T3E1 cells by enhancing LC3B-mediated autophagy through downregulation of miR-204. Experimental and therapeutic Medicine. 2020; 19: 883-890.

[26] Wang P, Zhu X, Yang L, Liang H, Feng S, Zhang R. Puerarin stimulates osteoblasts differentiation and bone formation through estrogen receptor, p38 MAPK, and Wnt/ $\beta$-catenin pathways. Journal of Asian Natural Products Research. 2012; 14 : 897-905.

[27] Wang Y, Wang WL, Xie WL, Li LZ, Sun J, Sun WJ, et al. Puerarin stimulates proliferation and differentiation and protects against cell death in human osteoblastic MG-63 cells via ER- dependent MEK/ERK and PI3K/Akt activation. Phytomedicine. 2013; 20: 787-796.

[28] Zhang Y, Zeng X, Zhang L, Zheng X. Stimulatory effect of puerarin on bone formation through activation of PI3K/Akt pathway in rat calvaria osteoblasts. Planta Medica. 2007; 73: 341-347.

[29] Liu L, Liu L, Bo T, Li S, Zhu Z, Cui R, et al. Puerarin Suppress Apoptosis of Human Osteoblasts via ERK Signaling Pathway. International Journal of Endocrinology. 2013; 2013: 1-6.

[30] Wang Y, Yang C, Xie WL, Zhao YW, Li ZM, Sun WJ, et al. Puerarin concurrently stimulates osteoprotegerin and inhibits receptor activator of NF-кB ligand (RANKL) and interleukin-6 production in human osteoblastic MG-63 cells. Phytomedicine. 2014; 21: 1032-1036.

[31] Tiyasatkulkovit W, Malaivijitnond S, Charoenphandhu N, Havill LM, Ford AL, VandeBerg JL. Pueraria mirifica extract and puerarin enhance proliferation and expression of alkaline phosphatase and type i collagen in primary baboon osteoblasts. Phytomedicine. 2014; 21: 1498-1503.

[32] Wang C, Meng M, Tang X, Chen K, Zhang L, Liu W, et al. The proliferation, differentiation, and mineralization effects of puerarin on osteoblasts in vitro. Chinese Journal of Natural Medicines. 2014; 12: 436-442.

[33] Wang XH, Shi XW, Luo XX, Zhang DH. Effect of puerarin on the proliferation and differentiation of osteoblasts and expression of type I collagen mRNA in a high-glucose environment. Acta Endocronologica. 2020; 16: 288-294.

[34] Zhang Y, Yan M, Yu Q, Yang P, Zhang H, Sun Y, et al. Puerarin Prevents LPS-Induced Osteoclast Formation and Bone Loss via Inhibition of Akt Activation. Biological \& Pharmaceutical Bulletin. 2016; 39: 2028-2035.

[35] Yang C, Li J, Zhu K, Yuan X, Cheng T, Qian Y, et al. Puerarin Exerts Protective Effects on Wear Particle-Induced Inflammatory Osteolysis. Frontiers in Pharmacology. 2019; 10: 1113.

[36] Lin S, Ke D, Lin Y, Fu X, Yu Y. Puerarin inhibits the migration of osteoclast precursors and osteoclastogenesis by inhibiting MCP-1 production. Bioscience, Biotechnology, and Biochemistry. 2020; 84: 1455-1459.

[37] Li J, Peng Y. Effect of puerarin on osteogenic differentiation of human periodontal ligament stem cells. Journal of International Medical Research. 2020; 48: 030006051985164.

[38] Wang Y, Yin L, Li Y, Liu P, Cui Q. Preventive effects of puerarin on alcohol-induced osteonecrosis. Clinical Orthopaedics and Related Research. 2008; 466: 1059-1067.

[39] Michihara S, Tanaka T, Uzawa Y, Moriyama T, Kawamura Y. Puerarin exerted anti-osteoporotic action independent of estrogen receptor-mediated pathway. Journal of Nutritional Science and Vitaminology. 2012; 58: 202-209.

[40] Tanaka T, Tang H, Yu F, Michihara S, Uzawa Y, Zaima N, et al. Kudzu (Pueraria lobata) vine ethanol extracts improve ovariectomy-induced bone loss in female mice. Journal of Agricultural and Food Chemistry. 2011; 59: 13230-13237.

[41] Liang J, Chen H, Pan W, Xu C. Puerarin inhibits caspase-3 expression in osteoblasts of diabetic rats. Molecular Medicine Reports. 2012; 5: 1419-1422.

[42] Luo Y, Zheng S, Ding Y, Dai Y, Zhou Y, Xiang R, et al. Preventive effects of kudzu root on bone loss and cartilage degradation in ovariectomized rats. American Journal of Translational Research. 2017; 9: 5180.

[43] Lim DW, Kim JG, Kim YT. Effects of dietary isoflavones from Puerariae radix on lipid and bone metabolism in ovariectomized rats. Nutrients. 2013; 5: 2734-2746.

[44] Tang W, Xiao L, Ge G, Zhong M, Zhu J, Qin J, et al. Puerarin inhibits titanium particle induced osteolysis and RANKL induced osteoclastogenesis via suppression of the NF $к B$ signaling pathway. Journal of Cellular and Molecular Medicine. 2020; 24: 11972-11983.

[45] Li B, Liu H, Jia S. Puerarin enhances bone mass by promoting osteoblastogenesis and slightly lowering bone marrow adiposity in ovariectomized rats. Biological \& Pharmaceutical Bulletin. 
2014; 37: 1919-1925.

[46] Lee M, Kim B, Lee Y, Park S, Shim J, Chung B, et al. Ameliorative Effects of Pueraria lobata Extract on Postmenopausal Symptoms through Promoting Estrogenic Activity and Bone Markers in Ovariectomized Rats. Evidence-Based Complementary and Alternative Medicine. 2021; 2021: 1-8.

[47] Cho HJ, Jun H, Lee JH, Jia Y, Hoang MH, Shim J, et al. Acute effect of high-dose isoflavones from Pueraria lobata (Willd.) Ohwi on lipid and bone metabolism in ovariectomized mice. Phytotherapy Research. 2012; 26: 1864-1871.

[48] Suthon S, Jaroenporn S, Charoenphandhu N, Suntornsaratoon P, Malaivijitnond S. Anti-osteoporotic effects of Pueraria candollei var. mirifica on bone mineral density and histomorphometry in estrogen-deficient rats. Journal of Natural Medicines. 2016; 70: 225-233.

[49] Yang X, Zhang H, Wang J, Zhang Z, Li C. Puerarin decreases bone loss and collagen destruction in rats with ligature-induced periodontitis. Journal of Periodontal Research. 2015; 50: 748757.

[50] Liu H, Li W, Jia S, Li B. Puerarin and zinc additively prevent mandibular bone loss through inhibiting osteoclastogenesis in ovariectomized rats. Histology and Histopathology. 2017; 32: 851-860.

[51] Yang X, Zheng H, Liu Y, Hao D, He B, Kong L. Puerarin for OVX-Induced Postmenopausal Osteoporosis in Murine Model: Systematic Review and Meta-Analysis. Current Stem Cell Research \& Therapy. 2020; 15: 37-42.

[52] Li B, Liu M, Wang Y, Gong S, Yao W, Li W, et al. Puerarin improves the bone micro-environment to inhibit OVX-induced osteoporosis via modulating SCFAs released by the gut microbiota and repairing intestinal mucosal integrity. Biomedicine \& Pharmacotherapy. 2020; 132: 110923.

[53] Urasopon N, Hamada Y, Asaoka K, Cherdshewasart W, Malaivijitnond S. Pueraria mirifica, a phytoestrogen-rich herb, prevents bone loss in orchidectomized rats. Maturitas. 2007; 56: 322-331.

[54] Xiao L, Zhong M, Huang Y, Zhu J, Tang W, Li D, et al. Puerarin alleviates osteoporosis in the ovariectomy-induced mice by suppressing osteoclastogenesis via inhibition of TRAF6/ROSdependent MAPK/NF-кB signaling pathways. Aging. 2020; 12: 21706-21729.

[55] Guo C, Xie J, Hong R, Pan H, Zhang F, Liang Y. Puerarin alleviates streptozotocin (STZ)-induced osteoporosis in rats through suppressing inflammation and apoptosis via HDAC1/HDAC3 signaling. Biomedicine \& Pharmacotherapy. 2019; 115: 108570 .
[56] Kim SY, Lee HJ, Kim T, Lee YG, Kwon JE, Kang SC. AntiOsteoporotic Activity of Pueraria lobata Fermented with Lactobacillus paracasei JS1 by Regulation of Osteoblast Differentiation and Protection against Bone Loss in Ovariectomized Mice. Fermentation 2021; 7: 186.

Abbreviations: ALP, Alkaline phosphatase; BAP, Bone alkaline phosphatase; BMC, Bone mineral content; BMD, Bone mineral density; BV/TV, Bone surface/bone volume; BS, Bone surface; Ct, Cortical bone tissue; CTx, Cterminal telopeptide; Ca, calcium; DPD, Deoxypyridinoline; EE, 17 alpha-ethinylestradiol; FM, Femoral metaphysis; FD, Femoral diaphysis; L4, Fourth lumbar vertebra; LPS, Lipopolysaccharides; MCF-7 cell, epithelial luminal cell line; MMP, Matrix metalloproteinase; NFATc1, Nuclear factor of activated T-cell, cytoplasmic 1; Ob.S, Osteoblast surface; Oc.S, Osteoclast surface; OVX, ovariectomized group; ORX, orchidectomized; OPG, Osteoprotegerin; OPN, Osteopontin; PPAR $\gamma$, Peroxisome proliferatoractivated receptor $\gamma$; PTIF, total isoflavones from Pueraria lobata; PVEE, kudzu vine ethanol extracts; RANKL, receptor activator of nuclear factor- $\kappa \mathrm{B}$ ligand; Tb, Trabeculars; Tb.N/Tb.Tn, Trabecular number; Tb.Sp, Trabecular separation; TD, Tibial diaphysis; TM, Tibial metaphysis; TRAP, Tartrate resistant acid phosphatase.

Keywords: Isoflavones; Puerarin; Bone; Osteoporosis; Pueraria lobata

Send correspondence to: Anna Gramza-Michałowska, Department of Gastronomy Sciences and Functional Foods, Faculty of Food Science and Nutrition, Poznań University of Life Sciences, 60-624 Poznań, Poland, E-mail: anna.gramza@up.poznan.pl 